\title{
Benefits of an Electronic Medical Records System in Rural Nepal
}

\author{
Watkinson-Powell $A,{ }^{1}$ Lee $A^{2}$ \\ 'Sheffield Medical School, Beech Hill Rd, Sheffield, South Yorkshire S10 2RX, UK, ${ }^{2}$ School of Health and Related Research, \\ The University of Sheffield, Western Bank, Sheffield, South Yorkshire S10 2TN, UK.
}

\section{ABSTRACT}

Introduction: Electronic medical records systems may improve the efficiency and quality of health services in developing countries. However the supporting evidence is limited as there are a number of barriers to their implementation, including lack of infrastructure, resources and skills. The objective of this study was to evaluate the introduction and assess the potential benefits of an Electronic Medical Records System in Rural at an NGO-supported health post in rural Nepal.

Methods: Original research in the form of a case report was carried out using participant observation of health clinics, semi-structured interviews with health workers and recording of time spent on clinic activities at the pilot and a control site.

Results: This evaluation found that the Electronic Medical Records System was well used and easy to learn. Health workers thought it improved continuity of care and found decision-support tools useful. Monthly report creation was faster but the system was difficult to integrate with government services, which limited the reduction in paperwork. Other problems identified included minor software issues, a lack of back-up, continuity of power supply and inadequate technical support. There was no significant impact on consultation length or time spent interacting with patients.

Conclusions: The introduction of the Electronic Medical Records System was largely successful. With adequate technical support and training, Electronic Medical Records System could provide a relatively low-cost means of improving patient care and health worker efficiency in developing countries. However they must be designed to fit their intended environment.

Keywords: computerized; electronic health records; medical records; medical records systems..

\section{INTRODUCTION}

The patient record is an essential tool for health workers to ensure both the quality and safety of patient care. Electronic medical records are widely adopted by healthcare providers in many developed countries. They provide health workers with a complete and rapidly accessible record of patient data and can help improve the efficiency of care delivery and lead to potential cost savings. ${ }^{1,2}$

The potential efficiency and quality improvement

Correspondence: Ms. Anna Watkinson-Powell, Sheffield Medical School, Beech Hill Road, Sheffield, S10 2RX, UK. Email: a.watkinsonpowell@googlemail.com, Phone: +7743132595. 
benefits of Electronic Medical Records System (EMRS) could help achieve better health care in the developing world. ${ }^{3}$ They can reduce medical errors, facilitate health worker decision making, enable faster report creation and improve disease monitoring. ${ }^{4-7}$ However, there are also many barriers to EMRS in these settings, including low levels of computer literacy, lack of infrastructure and resistance from health professionals. ${ }^{1,8,9}$ It would be difficult to justify investment of scarce resources in an EMRS until there is a more substantial body of evidence of their effectiveness. ${ }^{4}$

This study sought to evaluate the introduction of a purpose-designed EMRS at an NGO-supported health post in rural Nepal and to describe its initial impact on the quality and efficiency of patient care.

\section{METHODS}

This original case report is an evaluation of the PHASE Worldwide EMRS that was carried out one month after its introduction. PHASE Worldwide is a non-governmental organisation that supports and provides primary health care to eleven rural Village District Communities in Nepal. ${ }^{10}$ Each health post has a PHASE health worker, trained to the level of auxiliary nurse midwife. They provide outpatient and maternal health care services at sub-health posts and outreach clinics. Hagam was chosen as the pilot site due to its accessibility that allowed for easier external support and reliability of electricity supply. It is located in the Sindhupalchowk district of central Nepal. Fulpingkot was chosen as the control comparison site for pragmatic reasons due to its proximity to Hagam; it uses only paper records.

In 2010, PHASE designed an EMRS to overcome some of the problems with the existing paper-based recording system. These included an excessive amount of time taken to complete monthly reports and a lack of complete patient records that affected the ability to deliver continuity of care. PHASE piloted the use of EMIS $^{\circledR}$, a provider of EMRS software in primary health care in the UK. ${ }^{12}$ However, it proved too complex for the health workers. ${ }^{13}$ Subsequently, a bespoke EMRS was developed through a collaboration of PHASE health workers, UK doctors with past experience working for PHASE in Nepal, and computer sciences students at the University of Sheffield. An initial pilot was undertaken in 2010 following which some software revisions were made. The revised EMRS was introduced to the Hagam health post in December 2011. Staffs were provided with one day of training with the software.

The PHASE EMRS is a stand-alone system designed with a web interface. It consists of four key functions: patient registration, consultation records, a disease, drug and immunisation (D.D.I.) database and report creation. Once registered, each patient has a unique record of their demographic details and consultations. Decision-support tools include examination templates, immunisation alerts and allergy reminders, as well as links to PHASE's clinical guidelines and drug formulary. Consultations are recorded by the health worker at the point of care. Diagnoses and prescriptions are selected from lists of ICD-coded diseases and PHASE-specific drugs, with links to disease and drug information held on the database. The drug prescribed is automatically subtracted from the stock levels, which can also be edited manually. Monthly reports on clinic activities, morbidity and stock can be quickly generated and exported.

The evaluation used a mixed methods approach to gain a broader understanding of the success of implementation. Semi-structured interviews were conducted with the health workers at each site and participant observation was carried out during two clinic sessions in Fulpingkot and three in Hagam.

The fieldwork was carried out by a non-Nepali research student, independent and external to PHASE to help minimize potential observer bias. Face-to-face interviews were conducted in English with the two PHASE health workers in Hagam and Fulpingkot. Both health workers were female, aged 20-29 and trained as Auxiliary Nurse Midwives, typical of most PHASE health workers. Quantitative data was also collected at both sites on the length of consultations as well as the duration of time spent on paperwork and on the computer during consultations.

The data from the interviews and observation were descriptively summed up and analysed by identifying the key strengths and weaknesses of EMRS and highlighting common topics and concerns expressed by the health workers. The quantitative data were compared using two-tailed unpaired Student's t-test to identify any significant differences in the length of consultations and the time spent interacting with patients.

The health workers gave written informed consent to participate. Ethical approval was not required as this was an evaluation of an existing service.

\section{RESULTS}

The main benefit of the PHASE EMRS was faster completion of monthly reports, taking only a few minutes compared to 5-6 days previously. The staff also thought stock keeping would be less time consuming using the computer system, as the paper system took 2-3 hours after every clinic session. After one month, 
the Hagam health worker was observed to be able to accurately register patients, record consultations and create reports. Despite poor computer literacy, the health worker found the computer system relatively easy to learn, particularly as the reports were similar in format to the paper-based ones.

A number of technical issues were identified with the software, including how diseases were counted in the morbidity reports, an inability to edit consultations retrospectively and the lack of an 'other' diagnosis category. A few software functions were also not used at all, such as the "patient allergies" field or "immunisations", although the latter is currently the responsibility of state services. In addition, the health workers did not know how to export the reports into Excel or save them and there was no back-up system for the data held on the computer. The electricity supply in Hagam was relatively reliable during the study period but in Fulpingkot, it was erratic and unpredictable, with several power cuts observed. However, electricity was available for a sufficient amount of time to keep the laptop charged. There was also a lack of clarity as to how the monthly reports would be collected, as there are no printing or internet facilities in the villages and therefore no means of transmitting the information electronically. The health worker felt she would need more on-going support as problems arose.

There were problems integrating the EMRS with the paper reports required for government monitoring and with differentiating between patients seen by PHASE health workers and those from the government. As a result, the health worker was completing both paper and computer records. During the observation period the clinics saw few patients and all paper and computer records were completed during the clinics. However during busy clinics the health worker only used paper during the consultation, completing the computer record after the clinic. Paper morbidity reports had to include both all patients, whereas the reports generated on EMRS were limited to PHASE patients only.

The Hagam health worker thought EMRS enabled her to provide better patient care as she could assess responses to treatments, look for drug side effects and monitor changes in measurements such as blood pressure. In addition, the computer record contained more information about consultations than the paper registers. During the period of observation, seven out of the 29 patients (24\%) had an existing record on the computer. However it was observed that the staff rarely accessed previous consultations.

The health workers found the pre-programmed examination templates useful, particularly for antenatal care and paediatric visits, as they helped in the recording of patients' measurements. The health worker routinely used the template during consultations but they did not always complete all the data-entry fields. The inclusion of built-in questions also helped trigger health interventions; for example, one template had a question about immunisation status, which prompted staff to advise patients to attend a government immunisation clinic if necessary.

Although it was considered by staff to be much easier and quicker to look up the pre-programmed disease and drug information with the EMRS, during observation, the health worker rarely used this function. This was because they tended to see common ailments that did not require it. They did use the drug information to find paediatric drug doses.

The mean length of each consultation with EMRS in Hagam was 11.7 minutes. This was slightly longer than without EMRS in Fulpingkot ( $M=11.0$ mins) but the difference was not statistically significant $(P=0.509$, $95 \% \mathrm{Cl}-1.46$ to 2.91 ) (Figure 1 ). A slightly lower proportion of time in Hagam ( $M=54.4 \%$ ) was spent interacting with patients than in Fulpingkot ( $\mathrm{M}=60.3 \%)$ but this was not significant $(P=0.069, \mathrm{Cl}-12.20$ to 0.47). Out of total consultation time $39.7 \%$ (mean 4.2 minutes) was spent completing paperwork in Fulpingkot. With EMRS in Hagam, $12.5 \%$ of consultation time ( $\mathrm{M}=1.4 \mathrm{mins}$ ) was spent on paperwork and $33.1 \%$ on the computer $(\mathrm{M}=3.8 \mathrm{mins})$. The mean amount of time spent on the computer with EMRS (3.8mins) was not significantly different from that spent on paperwork without EMRS (4.2mins; $P=0.50$ ).

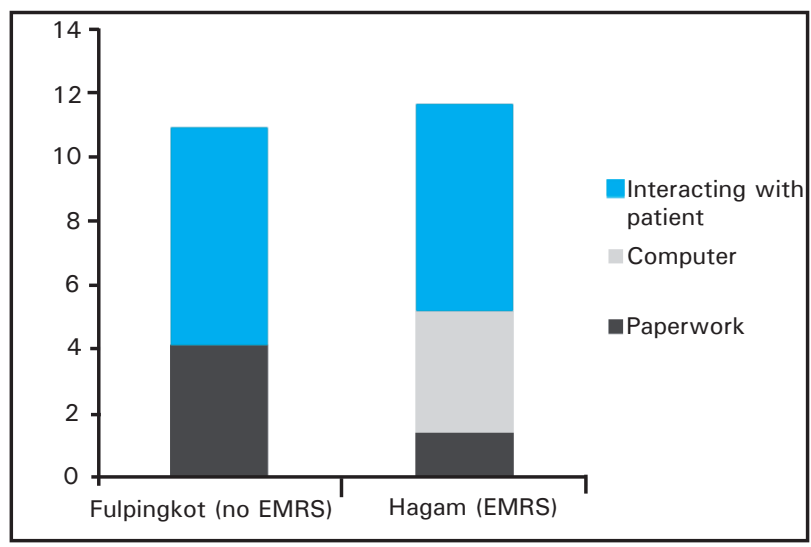

Figure 1. Mean amount of time in each consultation spent on paperwork, on the computer and interacting with the patient in Fulpingkot and Hagam

\section{DISCUSSION}

The introduction of the PHASE EMRS had some 
immediate and observable benefits. It saved time in report creation, consultation recording and stock keeping. The immediate availability of a complete patient record through EMRS may improve health worker decision making by allowing them to track responses to treatments and increasing continuity of care..$^{1,2}$ Of note, prior to EMRS there were no individual patient records. Although follow-up patients returning to a clinic were encouraged to bring their original outpatient tickets, many did not do this. EMRS can also reduce record duplication, allow faster and more accurate report generation and enable better data analysis. ${ }^{5,13}$ The quality and safety of care is also likely to improve with staff making greater use of features such as the drug information database to guide care.

Despite having these functionalities available, we found staff rarely referred to past patient records. This could be due to unfamiliarity with the system and current practice working without them. Whilst the availability of patients' records can improve continuity of care, a culture change in work practices is therefore needed to ensure that the staffs take full advantage of the range of functionalities offered. There was also a need for more support, an adequate back-up system and reliable electricity source. Further, work is also needed to resolve interface issues between national health information collection requirements and organizational needs.

The use of EMRS in developing countries is still in its infancy. In Nepal, some NGOs have started introducing such systems. The Child Welfare Scheme Nepal uses a custom-built EMRS at its Asha Healthcare clinic in Pokhara, although this is limited to a single urban centre (Andrew Clarke 2012, oral communication, 25 January).

Studies of EMRS implementation in developing countries have found the lack of infrastructure and computer literacy to be significant barriers to implementation. ${ }^{7,14}$ Failure is often due to poor design, lack of support after implementation and over-reliance on external resources. ${ }^{1-3,9}$ However, this study found that an EMRS can function well in a remote, resource-poor setting by tailoring the design to the intended context. ${ }^{7,13}$ Poor computer literacy need not preclude health workers from utilising an EMRS. PHASE designed a userfriendly interface and based the EMRS on the format of existing paper records, which has been shown to make computer systems easier to learn. ${ }^{4,13}$ There was no evidence that EMRS distracts the attention of health workers away from patients, as suggested elsewhere. ${ }^{13}$ The increase in consultation length was small and non- significant and data entry is likely to become faster as staff become more familiar with the system.

It is debatable whether EMRS is a priority in resourceconstrained countries struggling to provide universal health services. ${ }^{13}$ That said, the opportunities and benefits presented by this technology are considerable. Improving the continuity of care is key to the management of chronic non-communicable diseases and an electronic medical record greatly facilitates this. ${ }^{14}$ Furthermore, an EMRS could be particularly useful in remote settings where there is insufficient space and staff to support paper-based records. By saving considerable time in report creation, EMRS could increase efficiency and make better use of human resources. ${ }^{6}$ However, duplication of paper recording must be avoided and consideration given to how an EMRS is integrated with other existing health information systems. The aspiration ultimately is to develop and implement simple robust systems that necessitate minimal resource requirements and on-going technical support.

We considered the possibility of the "Hawthorne effect" with staff altering their behaviour in the presence of an observer. However, it was not possible to eliminate this. We also considered language barriers as a potential issue but found the health workers had sufficient spoken English to communicate effectively. The small sample and lack of randomisation in site selection may limit the generalizability of the results due to inherent variations between health posts and staff. However, the setting for this evaluation was significantly remote and rural, where implementation challenges are likely to be greater than elsewhere. We believe the evaluation demonstrates that the implementation of EMRS is feasible. Initial findings suggest improvements in care are likely but longer-term follow-up is needed to evaluate the long-term impact of EMRS on outcomes such as immunisation coverage and adverse drug reactions.

\section{CONCLUSION}

Electronic medical record systems can be implemented even in challenging settings and could provide a relatively low-cost means of improving patient care and reducing the workload of health workers. Challenges such as the lack of infrastructure and low computer literacy can be overcome if systems are well-designed and tailor-made to fit their intended environment.

\section{ACKNOWLEDGEMENTS}

We would like to acknowledge Gerda Pohl and all staff at PHASE Nepal. 


\section{REFERENCES}

1. World Health Organisation. Electronic Health Records. Manual for Developing Countries. [Online]. 2006 [cited 2012 Feb 2]; Available from: URL:http://www.wpro.who.int/ publications/docs/EHRmanual.pdf

2. Sandiford P, Annett H, Cibulskis R. What can information systems do for primary health care? An international perspective. Soc Sci Med. 1992;34(10):1077-87.

3. Scholl J, Syed-Abdul S, Ahmed LA. A case study of EMR system at a large hospital in India: challenges and strategies for successful adoption. J Biomed Inform. 2011;44(6):958-67.

4. Were M, Shen C, Bwana M, Emenyonu N, Musinguzi N, Nkuyahaga F, et al. Creation and evaluation of EMR-based paper clinical summaries to support HIV care in Uganda, Africa. Int J Med Inform. 2010;79(2):90-6.

5. Rotich JK, Hannan TJ, Smith FE, Bii J, Odero WW, Vu N, et al. Installing and implementing a computer-based patient record system in Sub-Saharan Africa: the Mosoriot Medical Record System. J Am Med Inform Assoc. 2003;10(4):295-303.

6. Tierney W, Rotich J, Smith F, Bii J, Einterz R, Hannan T. Crossing the 'digital divide': implementing an electronic medical record system in a rural Kenyan health center to support clinical care and research. Proc AMIA Symp. 2002:792-5.

7. Fraser H, Biondich P, Moodley D, Choi S, Mamlin BW, Szolovits P. Implementing electronic medical record systems in developing countries. Inform Prim Care. 2005;13(2):83-95.

8. Williams F, Boren SA. The role of electronic medical record in care delivery in developing countries. Int J Inf Manage. 2008;28:503-7.

9. Lucas H. Information and communications technology for future health systems in developing countries. Soc Sci Med. 2008;66:2122-32.

10. PHASE Worldwide. Phase Health Programmes. [Online]. [cited 2012 Feb 3]. Available from: URL: http://www. phaseworldwide.org/index.html

11. Egton Medical Information Systems Ltd. (EMIS). Company Profile. [Online]. [cited 2012 Feb 10]. Available from: URL:http://www.emis-online.com/company-profile

12. Cawley S, Cawley L. EMIS web pilot-report. 2010. Unpublished Report.

13. Kamadjeu R, Tapang E, Moluh R. Designing and implementing an electronic record system in primary care practice in sub-Saharan Africa: a case study from Cameroon. Inform Prim Care. 2005;13:179-86.

14. Littlejohns P, Wyatt J, Garvican L. Evaluating computerised health information systems: hard lessons still to be learnt. BMJ. 2003;326:850-3.

15. World Health Organisation. Global status report on non-communicable diseases 2010 [Online]. 2011. [cited 2012 Feb 16]. Accessible from: URL:http://www.who.int/nmh/ publications/ncd_report2010/en/ 